\title{
Interdisziplinäre Vertrauensforschung als Ort disziplinärer Selbstklärung
}

Marcel Egli

Die folgenden Überlegungen sind der Versuch, Form und Gehalt interdisziplinärer Vertrauensforschung $\mathrm{zu}$ verstehen. Solche Forschung bezeichnet eine Gesprächssituation, an der mehrere Vertreter disziplinärer Vertrauensforschung mitwirken. Unter disziplinärer Forschung wird eine Untersuchung mit dem Ziel nicht bloßer Addierung von Beschreibungen von Vorkommen von Vertrauen, sondern ihrer systematischen Darstellung in einer in sich kohärenten Vertrauenstheorie verstanden. Selbstverständlich müssen für diese Systematisierung Beschreibungen zur Kenntnis genommen werden; diese können jedoch nicht ohne Transformation und Entstellung der diese Beschreibungen leitenden Kriterien in die beabsichtigte Vertrauenstheorie eingehen.

Die These der Überlegungen ist dreiteilig. Sie lautet, a) dass das zu Klärende, Vertrauen, selbst zu seiner Klärung in Anspruch genommen wird. Die Klärung des Vertrauensthemas setzt also Vertrauen nicht nur als Forschungsimpuls voraus, sondern Vertrauen ermöglicht, begleitet und stützt auch seine eigene Klärung. Das zu Klärende ist im Klärungsvorgang selbst wirksam und nicht von ihm ablösbar. Deshalb können b) die disziplinären Teilnehmer in der mitVertrauen befassten interdisziplinären Arbeit gemeinsam weder auf den selben Gegenstand bezogen sein, noch ein Verständnis des Gegenstandes teilen, sondern sind aufgrund des an spezifisches Vertrauen gebundenen Zugangs in ihrem Verstehen mit einem Gegenstand befasst, der nur je in und mit diesem Zugang gegeben ist. Deshalb ist zudem c) das eigentliche Thema interdisziplinärer Arbeit zum Vertrauensthema nicht das, was - disziplinär - zur Klärung Anlass gibt (ein bestimmtes Vertrauen) oder das, was aus dieser Klärung - disziplinär - hervorgeht (ein bestimmtes Vertrauensverständnis), sondern letztlich bloß die einzelnen disziplinären Klärungsvorgänge als solche und die einzelnen disziplinären Selbstverständnisse hinsichtlich der jeweiligen Methode, die für die disziplinären Klärungsvorgänge (nicht nur, aber auch hinsichtlich Vertrauen) grundlegend ist. Erläuterung der These: 
a) Das zu Klärende wird zu seiner Klärung in Anspruch genommen:

$\mathrm{Zu}$ forschen heißt, zu versuchen, sich gegenüber dem, was einen umgibt, zu orientieren und Zusammenhänge zwischen dem, was einen umgibt, $\mathrm{zu}$ erkennen und $\mathrm{zu}$ einem Verstehen dieser $\mathrm{Zu}-$ sammenhänge zu gelangen. Dies, was einen umgibt, wird «der Gegenstand, im Sinne von (Forschungsinteresse bzw. verstandener Forschungsgegenstand, genannt.

Forschend verfügt man jedoch nicht im Voraus selbst über Gegenstandserkenntnis, sondern kommt zur Erkenntnis erst von dem Gegenstand her, auf den man sich bezogen findet. Dieses Bezogenwerden auf einen Gegenstand durch den Gegenstand ist Vertrauen.

Vertrauen ist in der Vertrauensforschung sowohl das, was zur Untersuchung steht, als auch das, was das, was zur Untersuchung steht, erschließt. Wenn Vertrauen erforscht und zu verstehen gesucht wird, gilt deshalb: Vertrauen ist und wird nie ein Gegenstand, auf den man sich - auswählend - beziehen kann, sondern Vertrauen bleibt schlicht das Bezogenwerden auf einen Gegenstand durch diesen Gegenstand.Vertrauen vollzieht sich, ohne zu einem Wissen zu werden. Ein Wissen von Vertrauen gibt es nur als angemaßtes Wissen. Vertrauen gibt es nur, indem es sich selber als solches erschließt. Aber es erschließt sich nur, indem es anderes erschließt.

b) Der verstandene Gegenstand ist nur je in und mit dem entsprechenden Zugang gegeben:

Nicht Vertrauen selbst kann also zur Sprache kommen, sondern nur die für eine Disziplin interessanten Gegenstände, Kontexte und Verfahren, durch, in und bei denen sich Vertrauen disziplinär erschlossen hat. Dieses Erschließungsverfahren ist die Methode der Disziplin. Die Gegenstände, Kontexte und Verfahren sind die Konstellation, die als Disziplin zu bezeichnen ist.

Die mit der Zeit etablierte Methode kann auch als Vertrautheit mit dem Gegenstand bezeichnet werden. Diese Vertrautheit besteht ausschließlich aufgrund des Gegenstandsbezugs durch den Gegenstand.

Die Klärung der Methode, in deren Verlauf dieser Vertrautheit nachgedacht wird, führt zur Beschreibung des grundlegenden Verfahrens der Disziplin und damit zur Klärung des disziplinären Selbstverständnisses.

c) Interdisziplinäre Arbeit ist der Diskurs über disziplinäre Selbstverständnisse:

Durch die Klärung der Methode wird ein Methodendiskurs ermöglicht. Er abstrahiert notwendigerweise vom Bezogenwerden auf 
einen bestimmten Gegenstand und thematisiert ausschließlich die Methode.

Dieses abstrakte Gespräch über die Methode kann auch mit jenen geführt werden, denen der konkrete Bezug zum Gegenstand fehlt.

Interdisziplinäre Arbeit ist ein solches abstraktes Methodengespräch über unterschiedliche Methoden.

Die Eigenheit einer Disziplin zeigt sich (in der Außenperspektive der beteiligten Disziplinen) darin, dass sich eine Disziplin von anderen Disziplinen in ihrer Methode unterscheidet. Die Klärung besteht dabei jedoch nicht darin, dass die in Frage stehende Disziplin ihren Platz in einer sie und die anderen Disziplinen leitenden und lozierenden Ordnung findet, sondern darin, dass sie erst durch das Gespräch an einen Ort gegenüber den am Gespräch beteiligten übrigen Disziplinen zu stehen kommt.

Disziplinäre Selbstklärung besteht aber eigentlich (in der Innenperspektive der Disziplin) im Beschreiben des für die Disziplin eigentümlichen Bezogenwerdens auf einen Gegenstand durch diesen Gegenstand. Gegenüber anderen Disziplinen unterscheidet sich die Disziplin nun durch das, worauf sie ihrerseits bezogen wird. Eine Disziplin hat dabei ihre Eigenheit nicht allein darin, dass sie bloß etwas in anderer Weise beschreibt, sondern vielmehr darin, dass sie faktisch anderes beschreibt.

$\mathrm{Ob}$ es in der interdisziplinären Arbeit immer um denselben Gegenstand geht oder nicht, lässt sich methodisch nicht klären, sondern ist Vertrauenssache, die der Reflexion bedarf, um Erkenntnis zu werden, die ihrerseits nicht im konkreten Gegenstandsbezug, sondern bloß abstrakt als disziplinäre Methode interdisziplinär diskutabel ist.

Zusammenfassend: In interdisziplinärer Perspektive gibt es Vertrauen nicht, sondern Vertrauen fungiert dabei schlicht als Zugehörigkeitsanzeige. Interdisziplinäre Vertrauensforschung befasst sich deshalb nicht mit Vertrauen als einem vorhandenen Gegenstand, sodass sie zu Wissen oder einem Zuwachs an Wissen über Vertrauen führen würde, sondern verdankt sich der disziplinären Selbsterschließung von Vertrauen durch Vertrauen. Das interdisziplinäre Gespräch fordert die beteiligen Disziplinen heraus, je Rechenschaft abzulegen über die Art und Weise, wie sich ihnen Vertrauen erschließt. Interdisziplinäre Vertrauensforschung gibt folglich ihren Akteuren zu verstehen, welche Disziplin womit und auf welche Weise befasst ist. Die disziplinäre Selbstklärung im Rahmen interdisziplinärer Vertrauensforschung 
erfolgt schlicht dadurch, dass eine Disziplin anderen zeigt, dass sie mit anderem als diese befasst ist.

- Marcel Egli arbeitet im Rahmen des Projekts «Vertrauen verstehen» an einer Dissertation mit dem Thema «Vertrauen und geschenkte Identität. Vertrauen in theologischer Perspektive im Anschluss an die Versöhnungslehre Karl Barths». 